\title{
Effect of obesity on perioperative outcomes after laparoscopic hepatectomy
}

\author{
Seeyuen J. Lee ${ }^{1}$, Adam Hauch², Erica Kane ${ }^{2}$, Christoper DuCoin², Michael Darden ${ }^{3}$, Geoffrey Parker ${ }^{4}$, Emad Kandil ${ }^{2}$, \\ Joseph F. Buell,
}

${ }^{1}$ Departments of Surgery, Louisiana State University School of Medicine, New Orleans, LA 70112, USA

${ }^{2}$ Departments of Surgery, Tulane University School of Medicine, New Orleans, LA 70112, USA

${ }^{3}$ Department of Economics, Tulane University, New Orleans, LA 70112, USA

${ }^{4}$ Tuck School of Business Administration, Dartmouth College, Hanover, NH 03755, USA

Correspondence to: Prof. Joseph F. Buell, Tulane Transplant Institute, Department of Surgery, Tulane University School of Medicine, 1415 Tulane Ave, New Orleans, LA 70112, USA. E-mail: jbuell1@tulane.edu

How to cite this article: Lee SJ, Hauch A, Kane E, DuCoin C, Darden M, Parker G, Kandil E, Buell JF. Effect of obesity on perioperative outcomes after laparoscopic hepatectomy. Hepatoma Res 2016;2:323-7.

\section{Article history:}

Received: 26-08-2016

Accepted: 17-10-2016

Published: 13-12-2016

Key words:

Liver resection,

laparoscopy,

obesity

\begin{abstract}
\end{abstract}
\section{INTRODUCTION}

Obese patients have frequently been perceived as challenging operative candidates often believed to incur increases in complications. Obesity is not only an increasing problem in the United States, but worldwide. Over $35 \%$ of the American population is now obese, and with the introduction of the Western diet these figures are climbing in both Europe and Asia. ${ }^{[1,2]}$ Worldwide, the prevalence of obesity has nearly doubled between 1980 and $2008 \cdot{ }^{[3]}$ Despite the dramatic rise in obesity, few studies have carefully examined the impact of a laparoscopic approach on the surgical outcomes of liver resection in these patients. 
As early as 1972, a Veterans Affairs study demonstrated that obese patients have a significantly higher incidence of pre-operative co-morbidities, specifically hypertension and diabetes ${ }^{[4]}$ Lending to this fact obese patients subsequently had a higher incidence of post-operative complications, including atelectasis and wound infection. Not surprisingly, the incidence of wound infections were most pronounced in obese diabetics, placing them at three fold increasing risk of post-operative mortality. The power of this study was limited because only $5 \%$ of the study cohort meets criteria for obesity at that time. Unfortunately, in the subsequent three decades, the landscape of mean body mass index and the incidence of obesity has dramatically changed.

Dindo et al. ${ }^{[5]}$ first presented a classification system designed to identify and define post-operative complications. Their study examined and analyzed the outcomes of over six thousand open general surgery patients. This analysis identified the only increased complication in the obese patient was the rate of wound infections, and failed to identify an increased rate of any additional complications. This observation was not only true for the obese patient, but also for the severely obese patients with body mass index (BMI) greater than 35. In their final analysis they concluded and advocated for surgical intervention in the "rapidly expanding obese population".

Ironically this is in sharp contrast with the early National Institutes of Health guidelines for laparoscopy cholecystectomy that excluded the morbidly obese patients ${ }^{[6]}$ Despite this consensus guideline, surgeons quickly identified laparoscopy was an ideal approach for obese patients. Theoretically, laparoscopy lends to improved visualization, smaller incisions, and less physiologic impact. These advantages result in: (1) shorter hospital stays; (2) rapid return to normal diet; and (3) fewer complications. ${ }^{[7]}$ However, it would be decades before Tsinberg first examined the effect of obesity in a small cohort of minor laparoscopic hepatic resections. ${ }^{[8]}$ This, and subsequent studies, have confirmed the benefits of laparoscopy but only at the expense of increased operative times with the occurrence of Clavien-Dindo class I and II complications. $^{[9,10]}$ Our current study seeks to evaluate the effect of laparoscopy on a large group of open and laparoscopic minor and major resections.

\section{METHODS}

From January 2001 to September 2015, 640 patients underwent liver resection by a single surgeon. Of those, 396 patients underwent a laparoscopic hepatectomy. All patients were included in this study.
Patients were evaluated in 4 weight groups: normal (BMI < 25), overweight (BMI $\geq 25)$, obese $(\mathrm{BMI} \geq$ 30 ), and severely obese (BMI $\geq 35$ ). Based on World Health Organization (WHO) classifications of obesity, there were $78(20 \%)$ normal weight patients, 209 $(52 \%)$ overweight patients, $86(22 \%)$ obese, referred by the WHO as class I obesity, and $23(6 \%)$ severely obese, referred by WHO as class II and III obesity. ${ }^{[11]}$

Patient demographics, clinical status, tumor characteristics, operative and postoperative outcomes, as well as clinicopathologic data were analyzed among each weight class against the normal BMI group. The surgical technique of laparoscopic hepatectomy utilized by this surgeon has been well reported in the literature ${ }^{[12]}$ Laparoscopic hepatectomy was selectively performed with hand port assistance based on tumor location, accessibility and condition of the underlying liver parenchyma.

\section{Statistical analysis}

Continuous variables were compared between groups using Student's $t$-test; categorical variables were compared using chi-squared test. Serial values were compared using analysis of variance. A univariate model was used to identify all variables significantly associated with post-operative complications. To examine the effect of obesity on the laparoscopic approach a full cohort analysis of all resections was performed while a second analysis of only laparoscopic resections was carried out. A multivariate regression model was then developed to identify the independent variables that maintained significance in multivariate analysis. A $P$-value of $<0.05$ was considered statistically significant.

\section{RESULTS}

Several demographic differences were noted between the study groups. Mean age was similar, while severe obesity and malignant disease had a higher association with male gender. The incidence of comorbidities including hypertension and diabetes increased with increasing weight class. American Society of Anesthesiologists (ASA) score increased concordantly with patients' body weight. However, the incidence of cirrhosis, the number of segments resected and the percentage of major resections were similar across all weight groups [Table 1].

Outcome data identified an increase of blood loss, transfusions, and complications in patients moving from normal, overweight, obese and severely obese patients. Surgical margins were similar across groups. Clavien-Dindo I-V complications were significantly different between the severely obese and the other 
Table 1: Patient demographics and tumor characteristics

\begin{tabular}{|c|c|c|c|c|c|}
\hline & Normal (BMI < 25) & Overweight (BMI 25-30) & Obese (BMI 30-35) & Severely obese (BMI $\geq 35)$ & ANOVA $P$-value \\
\hline Number of patients & 78 & 209 & 86 & 23 & \\
\hline Median BMI & 23 & 27 & 30 & 40.5 & \\
\hline Age, years & 53.6 & $53.8(0.92)$ & $56.3(0.26)$ & $61.1(0.69)$ & \\
\hline Male gender & $28 \%$ & $36 \%(0.19)$ & $42 \%(0.07)$ & $65 \%(<0.01)$ & 0.03 \\
\hline ASA & 2.76 & $2.68(0.34)$ & $2.92(0.11)$ & $3.00(0.14)$ & $<0.01$ \\
\hline Hypertension & $42 \%$ & $40 \%(0.35)$ & $63 \%(<0.01)$ & $78 \%(<0.01)$ & $<0.01$ \\
\hline Type II DM & $12 \%$ & $10 \%(0.62)$ & $37 \%(<0.01)$ & $70 \%(<0.01)$ & $<0.01$ \\
\hline Cirrhosis & $22 \%$ & $12 \%(0.03)$ & $19 \%(0.61)$ & $13 \%(0.35)$ & 0.98 \\
\hline Segments & 2.3 & $2.5(0.22)$ & $2.3(0.89)$ & $2.5(0.39)$ & 0.92 \\
\hline Major & $24 \%$ & $23 \%(0.96)$ & $20 \%(0.62)$ & $17 \%(0.53)$ & 0.93 \\
\hline Malignancy & $47 \%$ & $40 \%$ & $50 \%$ & $57 \%$ & \\
\hline
\end{tabular}

Data in brackets represent $P$-values compared to normal. BMI: body mass index; ASA: American Society of Anesthesiologists; DM: diabetes mellitus; ANOVA: analysis of variance

Table 2: Intraoperative and postoperative data

\begin{tabular}{lcccc}
\hline & Normal (BMI < 25) & Overweight (BMI 25-30) & Obese (BMI 30-35) & Severely obese (BMI $\geq$ 35) \\
\hline Number of patients & 78 & 209 & 86 & 23 \\
OR time $(\mathrm{h})$ & 2.2 & $2.5(0.02)$ & $2.4(0.18)$ & $2.5(0.13)$ \\
EBL $(\mathrm{mL})$ & 177 & $234(0.05)$ & $165(0.95)$ & $254(0.22)$ \\
Transfusion & $6.4 \%$ & $7.1 \%(0.05)$ & $7.0 \%(0.08)$ & $8.7 \%(0.08)$ \\
Margin $(\mathrm{cm})$ & 1.1 & $1.0(0.38)$ & $1.0(0.40)$ & $1.2(0.65)$ \\
LOS (days) & 3.5 & $3.2(0.53)$ & $3.3(0.73)$ & $4.6(0.08)$ \\
Complication & $15.3 \%$ & $17.2 \%(0.71)$ & $18.6 \%(0.58)$ & $47.8 \%(0.01)$ \\
Deaths & $2.5 \%$ & $0.4 \%(0.12)$ & $1.1 \%(0.54)$ & $4.3 \%(0.66)$ \\
\hline
\end{tabular}

Data in brackets represent $P$-values compared to normal. BMI: body mass index; OR: operating room; EBL: estimated blood loss; LOS: length of stay

Table 3: Distribution of complications

\begin{tabular}{|c|c|c|c|c|}
\hline & Normal $(n=78)$ & Overweight $(n=209)$ & Obese $(n=86)$ & Severely obese $(n=23)$ \\
\hline Overall incidence & $12(15 \%)$ & $36(17 \%)$ & $16(19 \%)$ & $11(48 \%)$ \\
\hline Cardiac & $1(1.3 \%)$ & $2(0.9 \%)$ & $1(1.2 \%)$ & $1(4.3 \%)$ \\
\hline $\begin{array}{l}\text { Pulmonary } \\
\text { PE }\end{array}$ & $\begin{array}{r}2(2.6 \%) \\
0(0 \%)\end{array}$ & $\begin{array}{c}6(2.9 \%) \\
0(0 \%)\end{array}$ & $\begin{array}{l}2(2.3 \%) \\
2(2.3 \%)\end{array}$ & $\begin{array}{l}2(8.7 \%) \\
2(8.7 \%)\end{array}$ \\
\hline $\begin{array}{l}\text { Gastrointestinal } \\
\text { Bile leak }\end{array}$ & $\begin{array}{l}6(7.7 \%) \\
4(5.1 \%)\end{array}$ & $\begin{array}{c}10(4.8 \%) \\
5(2.3 \%)\end{array}$ & $\begin{array}{l}7(8.1 \%) \\
4(4.7 \%)\end{array}$ & $\begin{array}{c}4(17.4 \%) \\
3(13 \%)\end{array}$ \\
\hline Wound & $0(0 \%)$ & $4(1.9 \%)$ & $2(2.3 \%)$ & $1(4.3 \%)$ \\
\hline Other & $1(1.3 \%)$ & $15(7.1 \%)$ & $4(4.7 \%)$ & $1(4.3 \%)$ \\
\hline \multicolumn{5}{|c|}{ Percentage of Clavian-Dindo complications } \\
\hline 5 & $2(17 \%)$ & $1(3 \%)$ & $1(6.3 \%)$ & $1(9 \%)$ \\
\hline 4 & $1(8 \%)$ & $6(17 \%)$ & $4(25 \%)$ & $1(9 \%)$ \\
\hline 3 & $2(17 \%)$ & $17(47 \%)$ & $4(25 \%)$ & $1(9 \%)$ \\
\hline 2 & $2(17 \%)$ & $1(3 \%)$ & $1(6.3 \%)$ & $3(27 \%)$ \\
\hline 1 & $5(42 \%)$ & $7(19 \%)$ & $6(38 \%)$ & $5(45 \%)$ \\
\hline
\end{tabular}

Data are shown as $n(\%)$. PE: pulmonary embolism

BMI groups [Tables 2 and 3]. In the first regression analysis, we analyzed all hepatectomies $(n=640)$, both open and laparoscopic. Initial univariate analysis of factors associated with complications included open surgery, race, BMI, ASA, transfusion, operating room time, and major resection; however, the multivariate regression analysis resulted in only two significant factors: ASA $(P<0.001)$ and transfusion requirement $(P$ $<0.001)$. A subsequent analysis was performed on just the laparoscopic hepatectomy group $(n=396)$; where univariate analysis identified BMI, ASA, diabetes, transfusion, and major hepatectomy were associated with complications. After multivariate regression modeling, only ASA $(P<0.001)$ and transfusion requirement $(P<0.002)$ were again significantly associated with postoperative complications [Table 4].

\section{DISCUSSION}

Historically, obese patients have been perceived to incur poorer post-operative outcomes compared to normal body mass index patients. Initially this assumption was 
Table 4: Final regression models

\begin{tabular}{lcccc}
\hline \multirow{2}{*}{ Variables } & \multicolumn{2}{c}{$\begin{array}{c}\text { Univariate } \\
\text { analysis }\end{array}$} & \multicolumn{2}{c}{$\begin{array}{c}\text { Multivariate } \\
\text { regression }\end{array}$} \\
\cline { 2 - 6 } & $\boldsymbol{t}$-stat & $\boldsymbol{P}$-value & $\boldsymbol{t}$-stat & $\boldsymbol{P}$-value \\
\hline All open and laparoscopic liver resections $(n=640)$ & \\
Open surgery & -5.60 & $<0.001$ & & \\
Gender & 1.98 & 0.048 & & \\
Race & 3.47 & $<0.001$ & & \\
ASA & 6.29 & $<0.001$ & 5.07 & $<0.001$ \\
Obesity & 4.11 & $<0.001$ & & \\
Hypertension & 0.24 & 0.810 & & \\
Diabetes & 2.14 & 0.033 & & \\
INR & 1.57 & 0.117 & & \\
Cirrhosis & 1.71 & 0.242 & & \\
Major resection & 3.50 & $<0.001$ & & \\
OR time & 5.96 & $<0.001$ & & \\
EBL & 4.20 & $<0.001$ & & \\
Transfusion & 7.24 & $<0.001$ & 6.02 & $<0.001$ \\
Laparoscopic liver resections $(n=396)$ & & \\
Gender & 1.45 & 0.148 & & \\
Race & -0.20 & 0.842 & & \\
ASA & 3.11 & $<0.001$ & 3.45 & $<0.001$ \\
BMI & 2.18 & 0.029 & & \\
Hypertension & 0.32 & 0.749 & & \\
Diabetes & 2.24 & 0.026 & & \\
INR & 1.32 & 0.188 & & \\
Cirrhosis & 1.32 & 0.188 & & \\
Major resection & 2.17 & 0.031 & & \\
OR time & 0.81 & 0.418 & & \\
EBL & 2.30 & 0.022 & & \\
Transfusion & 2.75 & 0.006 & 3.10 & $<0.002$ \\
\hline
\end{tabular}

BMI: body mass index; ASA: American Society of Anesthesiologists; OR: operating room; EBL: estimated blood loss; INR: international normalized ratio

not only applied to open surgery but also laparoscopic surgery, considering the inherent technical challenges in the obese patient. However, laparoscopy has quickly become the favorable or even preferred approach for general surgery in obese patients including cholecystectomy, and colectomy. ${ }^{[13]}$ The current study examines a single surgeon's experience with laparoscopic and open hepatectomy in a broad group of obese and non-obese patients. Our hypothesis was to affirm the laparoscopic approach's viability as an alternative to open hepatectomy with respect to operative outcomes, including length of stay and the incidence and severity of complications.

In our analysis of the laparoscopic group, a higher incidence of complications was not identified until patients reached severe obesity. Univariate analysis of the entire group did identify obesity measured by $\mathrm{BMI}$ as a predictor of complications. However, under multivariate regression analysis, BMI lost significance and was no longer a predictor of complications in patients undergoing laparoscopic hepatectomy.
As a baseline the overweight, obese and severely obese patients in our study had a significantly higher incidence of co-morbidities including hypertension and type II diabetes resulting in higher ASA scores. This same increased incidence in co-morbidities and ASA scores did not result in longer operative times when comparing obese and severely obese patients to normal BMI patients.

Our patient cohort had several interesting trends that may have led to some bias including a higher incidence of male patients undergoing laparoscopic hepatectomy and the use of laparoscopic resection in males with malignant disease. This selection bias for males and malignant disease may have contributed to the severely obese patients incurring a higher incidence of complications. The distribution of complications defined by the Clavien-Dindo classification was similar across all BMI groups except the severe obese. In this group of severely obese there was a higher incidence of pulmonary complications. However, the low incidence of class III and IV complications was observed in the severe obese population, which may reflect the small study population or a selection bias.

Our final regression model identified ASA score and transfusion as the best associations with the occurrence of complications. The statistical model for complications increased with rising ASA scores. This positive predictor underscores the power and utility of ASA in clinical decision-making. Despite the presence of transfusion in the final model, its impact on complications may require further evaluation. Transfusion may be more complex variable than a measure of blood loss, the need for blood or blood products. Transfusion may serve also as a surrogate marker for a complex surgical patient with a multitude of inherent and underlying variables such as liver steatosis, functional hepatic reserve or even case complexity due to obesity.

In our experience, laparoscopic hepatectomy is a safe, effective procedure with complications rates and Clavien-Dindo severity scores comparable to open hepatectomy for most obese patients. The caveat to this statement is that in severely obese patients (BMI $>35$ ) there was significant rise in complications. This may reflect the effect of obesity or is a direct result of increasing patient ASA or even selection bias. This lends to the last question of does the underlying liver quality, most notably steatosis, contribute the incidence and severity of complications? This study however, reaffirms the belief that the benefits of laparoscopic hepatectomy apply to the overweight 
and obese patients, while identifying areas that warrant further studies.

\section{Financial support and sponsorship \\ None.}

\section{Conflicts of interest}

There are no conflicts of interest.

\section{Patient consent}

All patients gave their consent forms before treatment.

\section{Ethics approval}

The study was approved by the ethical review board of Tulane University.

\section{REFERENCES}

1. Flegal KM, Carroll MD, Kit BK, Ogden CL. Prevalence of obesity and trends in the distribution of body mass index among US adults, 1999-2010. JAMA 2012;307:491-7.

2. Finucane MM, Stevens GA, Cowan MJ, Danaei G, Lin JK, Paciorek CJ, Singh GM, Gutierrez HR, Lu Y, Bahalim AN, Farzadfar F, Riley LM, Ezzati M; Global Burden of Metabolic Risk Factors of Chronic Diseases Collaborating Group (Body Mass Index). National, regional, and global trends in body-mass index since 1980: systematic analysis of health examination surveys and epidemiological studies with 960 country-years and 9•1 million participants. Lancet 2011;377:557-67.

3. Obesity: preventing and managing the global epidemic. Report of a
WHO consultation. World Health Organ Tech Rep Ser 2000;894:i-xii, $1-253$.

4. Postlethwait RW, Johnson WD. Complications following surgery for duodenal ulcer in obese patients. Arch Surg 1972;105:438-40.

5. Dindo D, Muller MK, Weber M, Clavien PA. Obesity in general elective surgery. Lancet 2003;361:2032-5.

6. Gadacz, TR, Talamini MA. Traditional versus laparoscopic cholecystectomy. Am J Surg 1991;161:336-8.

7. Miles RH, Carballo RE, Prinz RA, McMahon M, Pulawski G, Olen RN, Dahlinghaus DL. Laparoscopy: the preferred method of cholecystectomy in the morbidly obese. Surgery 1992;112:818-22; discussion 822-3.

8. Tsinberg M, Tellioglu G, Simpfendorfer CH, Walsh RM, Vogt D Fung J, Berber E. Comparison of laparoscopic versus open liver tumor resection: a case-controlled study. Surg Endosc 2009;23:847-53.

9. Johnson LB, Graham JA, Weiner DA, Smirniotopoulos J. How does laparoscopic-assisted hepatic resection compare with the conventional open surgical approach? J Am Coll Surg 2012;214:717-23; discussion 723-5.

10. Viganò L, Kluger MD, Laurent A, Tayar C, Merle JC, Lauzet JY, Andreoletti M, Cherqui D. Liver resection in obese patients: results of a case-control study. HPB (Oxford) 2011;13:103-11.

11. Tanaka S, Iimuro Y, Hirano T, Hai S, Suzumura K, Nakamura I, Kondo Y, Fujimoto J. Safety of hepatic resection for hepatocellular carcinoma in obese patients with cirrhosis. Surg Today 2013;43:12907

12. Buell JF, Thomas MJ, Doty TC, Gersin KS, Merchen TD, Gupta M, Rudich SM, Woodle ES. An initial experience and evolution of laparoscopic hepatic resectional surgery. Surgery 2004;136:804-11.

13. Leroy J, Ananian P, Rubino, F, Claudon B, Mutter D, Marescaux $\mathrm{J}$. The impact of obesity on technical feasibility and postoperative outcomes of laparoscopic left colectomy. Ann Surg 2005;241:69-76. 